\title{
Vibration and pressure wave therapy for calf strains: a proposed treatment
}

\author{
Amol Saxena \\ Marie St. Louis \\ Magali Fournier
}

Department of Sports Medicine, Palo Alto Medical Foundation, Palo Alto, CA

Corresponding author:

Amol Saxena

Department of Sports Medicine

Palo Alto Medical Foundation

795 El Camino Real

Palo Alto, CA 94301

e-mail: Heysax@aol.com

\section{Summary}

Calf (lower leg) strains have a variety of treatment regimens with variable outcomes and return to activity (RTA) time frames. These injuries involve disruption of portions or the entire gastrocnemius-soleus myo-tendinous complex. Conservative treatment initially consists of rest, ice, compression, elevation (RICE). Immediately following calf injury, patients can utilize cryotherapy, massage, passive range of motion, and progressive exercise. In general, Grade I through Grade III calf strains can take up to 6 weeks before the athlete can return to training. It can also involve the loss of more than $50 \%$ of muscle integrity. Recently, vibration therapy and radial pressure waves have been utilized to treat muscular strains and other myo-tendinous injuries that involve trigger points. Studies have suggested vibration therapy with rehabilitation can increase muscle strength and flexibility in patients. Segmental vibration therapy (SVT) is treatment to a more focal area. Vibration therapy (VT) is applied directly to the area of injury. VT is a mechanical stimulus that is thought to stimulate the sensory receptors, as well as decrease inflammatory cells and receptors. Therefore, VT could be a valuable tool in treating athlete effectively and decreasing their recovery time. The purpose of this paper is to give the reader baseline knowledge of VT and propose a treatment protocol for calf strains using this technology along with radial pressure waves.

KEY WORDS: calf strain, radial pressure wave, vibration therapy.

\section{Introduction}

Calf (lower leg) strains have a variety of treatment regimens with variable outcomes and return to activity (RTA) time frames ${ }^{1-3}$. These injuries involve disruption of portions or the entire Gastrocnemius-soleus myo-tendinous complex. Rupture of the gastrocnemius region medially is termed "tennis leg"1. Conservative treatment initially consists of rest, ice, compression, elevation (RICE). NSAIDs can be utilized, though this is controversial ${ }^{1,2}$. Immediately following calf injury, patients can utilize cryotherapy, massage, passive range of motion, and progressive exercise. In general, Grade I injuries, which have partial tearing with no loss of muscle integrity, heal in a few days to a few weeks. Grade II muscle strains (10-50\% of muscle disruption with loss of strength) need one to 6 weeks before the patient can return to training. Grade III injuries could take up to six months for return to activity (RTA) as they involve loss of more than $50 \%$ of muscle integrity and have noticeable defects ${ }^{1}$. Reinjury is frustrating and common ${ }^{1-3}$. Recently, vibration therapy has been utilized to treat muscular strains and other myo-tendinous injuries that involve trigger points ${ }^{3-7}$.

Whole body vibration (WBV) has been used to increase neuromuscular activity through the use of mechanical activation. Studies have suggested vibration therapy with rehabilitation can increase muscle strength and flexibility in patients ${ }^{6,8}$. Segmental vibration therapy (SVT) is treatment to a more focal area ${ }^{3}$. Vibration therapy (VT) is applied directly to the area of injury. VT is a mechanical stimulus that is thought to stimulate the sensory receptors, as well as decrease inflammatory cells and receptors. The process by which this is accomplished is not yet understood. It has been proposed that VT helps muscle extensibility, decreasing muscle strain and possibly improve strength $^{3}$. The purpose of this paper is to educate the reader about VT and suggest a treatment protocol specifically for calf strains based on current research; they are common injuries and recovery can been frustrating with historic treatment regimens.

Proposed treatment protocol for calf strains:

The patient is positioned kneeling on a chair with their back to the provider, with the knee flexed. The V-Actor ${ }^{\circledR}$ device (Storz Medical AG Tägerwilen, Switzerland) is a SVT device which produces vibrations up to $35 \mathrm{~Hz}$. This device is applied to the affected area for 2500 pulses at 4.0 Bar and $35 \mathrm{~Hz}$ (Fig. 1). Following this, a radial pressure wave ("EPAT ${ }^{\circledR}$ ") de- 


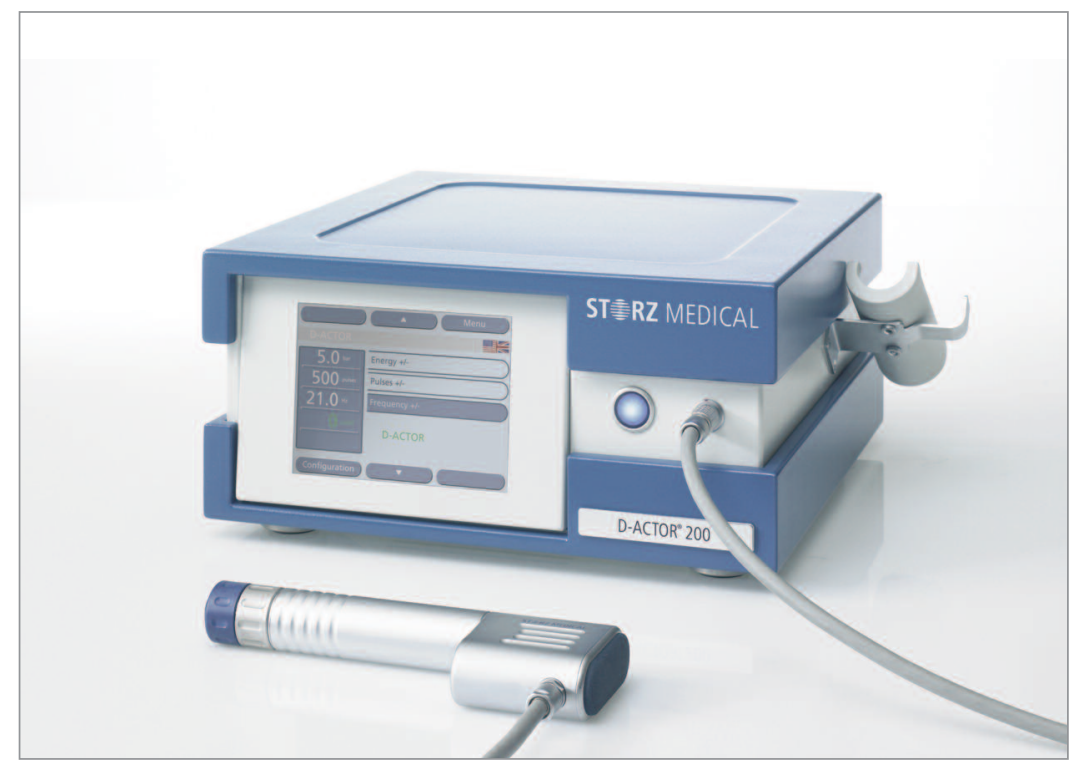

Figure 1. D-Actor 200 Storz AG.

vice, the D-Actor ${ }^{\circledR}$ (Storz Medical AG Tägerwilen, Switzerland) is used to treat the affected area for 2500 pulses at $4.0 \mathrm{Bar}$ and $11 \mathrm{~Hz}$. If patients cannot tolerate 4.0 Bar then we will start treatments at 3.0 Bar and gradually rise to 4.0 based on tolerance. These two types of treatments are able to be combined in one device. This pressure wave treatment typically takes four minutes, which corresponds with current research recommendations ${ }^{3,4,7}$.

Activities as tolerated are permitted after the treatments though generally running and jumping are not allowed for 3-6 weeks. Two additional treatments are administered at weekly intervals. These treatments can be repeated as needed. So far research does not show increased gain with SVT treatments more than 5 weeks with the frequency $35-50 \mathrm{~Hz}$ (set to the tolerance of the patient) ${ }^{3,4}$. Post-treatment soreness can occur. Other treatments such as strengthening, flexibility exercises and cryotherapy can be continued. NSAIDs are discouraged ${ }^{7,9}$.

\section{Discussion}

In 2010, Broadbent et al. reviewed the response of muscle inflammation and muscle soreness with $\mathrm{VT}^{4}$. This study had subject's quadriceps, calf, gluteals, and ITB treated with a VibroGym ${ }^{\circledR}$ vibration platform. The therapy was three sessions of one minute of vibration with 45 second of rest between the sessions with amplitude of $5 \mathrm{~mm}$ and $50 \mathrm{~Hz}$ frequency. Both control and vibration groups had to run 40 minutes three to four times a week prior to intervention. A visual analogue pain scale was used and blood was drawn at 24 hour intervals for five days. Both groups were not allowed to run during the five days of follow up. Soreness decreased more in the vibration treatment group by the fifth day as compared to the con- trol group. Findings also showed a decrease in IL6 at five days after an increase at the first 24 hours as compared to the control group. There was a decrease in CRP and Histamine at five days. Broadbent et al. related the CPK findings were unclear ${ }^{4}$.

Another consideration for SVT is the increase in muscle extensibility; this has been seen in a study from 2011 by Bakhtiary et al. ${ }^{5}$. This study investigated the flexibility of the hamstrings of 30 non-athletic females who had three sessions per week of SVT for eight weeks. Each session consisted of at least three sets of vibrations at $50 \mathrm{~Hz}$ for one minute with one minute rest between the treatments. Subjects were placed in a prone position with extension at the hip and knee joint with vibration therapy applied directly to the hamstring. Treatment was increased incrementally every 2 weeks. In the final 2 weeks sessions, four sets of vibration treatment were rendered, and the duration of vibration treatment was one minute. There was one minute rest between each application during all sessions. The study showed significant increases in flexibility in the group treated with vibration versus the conservative/stretching treatment ${ }^{5}$.

SVT for the lower extremity was reviewed by Peer et al. ${ }^{3}$. They investigated the effects of SVT on ankle sprains and hamstring injuries using the Swisswing ${ }^{\circledR}$. Ankles sprains were treated at three places on the lower leg: the bottom of the foot, heel and Gastrocnemius. Each region is placed on the drum for two minutes at $20 \mathrm{~Hz}$. Hamstring strains were treated in a standing and sitting position at $20 \mathrm{~Hz}$ for 2 minutes. Gluteals and hamstring are treated in a standing position with the muscle resting against the drum of the Swisswing ${ }^{\circledR}$. The gastrocnemius is treated in a sitting position, and the calf is resting over the drum. The control group was treated by conservative measures with rest, ice and compression. The Swisswing ${ }^{\circledR}$ treatment showed increase ROM at the ankle, and in- 
creased hamstring flexibility compared to the post control treatment as well as baseline. There was also a decrease in stiffness at the ankle as well as the hamstring after SVT. The recommended duration of the treatment was four minutes, and rest post-treatment is to patient tolerance ${ }^{3,4}$. Recent research on radial pressure waves for other musculoskeletal strains could be applied to calf strains ${ }^{9}$. The treatments with these types of modalities also last approximately four minutes.

Soreness from calf strain and delay of return to the activity can result in loss of fitness and strength. Some efficacy has been seen in treating multiple areas and sessions depending on the muscle group. Trigger points can be used as guides for determining the areas of treatment. This could be a helpful addition to getting athletes back into their sports faster. As noted by previous studies, SVT can be applied more frequently than traditional sound wave and ESWT treatments $3,5,7$.

Cacchio et al. reported on their results of a Level I study on "shockwave" for proximal hamstring tendinopathy. They compared a similar radial pressure wave device to standard physiotherapy for chronic hamstring tendinopathy in a randomized control trial. Eighty percent of those receiving radial pressure waves returned to sport by three months while $10 \%$ who had standard physiotherapy were able to do so ${ }^{10}$. One should look at their study as a good example to prepare a similar study for Gastrocnemius strains.

We propose a study to compare "traditional" treatment for a similar grade of Gastrocnemius strain with the application of SVT and radial pressures waves. One should document pre- and post-treatment VAS, activity level, RTA and ROM of the ankle and knee. A standardize protocol should be followed and then subsequently evaluated using evidenced-based methods. Segmental vibration therapy combined with radial pressure waves, is a new modality that research may reveal useful in treating calf injuries. Further study needs to be performed with SVT combined with radial pressure waves for muscle injury.

\section{References}

1. Dixon JB. Gastrocnemius vs. Soleus Strain: How to Differentiate and Deal with Calf Muscle Injuries. Curr Rev Musculoskelet Med 2009 June; 2(2):74-77.

2. Glazer JL, Hosey RG. Soft-Tissue Injuries of the Lower Extremity. Prim Care Clin Office Pract 2004; 31:1005-1024.

3. Peer K, Barkley J, Knapp D. The Acute Effects of Local Vibration Therapy on Ankle Sprain and Hamstring Strain Injuries. Physician and Sports Medicine 2009; 4(37):1-8.

4. Broadbent S, Rousseau J, J. Throp RM, Choate SL, Jackson FS, Rowlands DS. Vibration Therapy Reduces Plasma IL6 and Muscle Soreness After Downhill Running. Br J Sports Med 2010; 44:888-894

5. Bakhtiary AH, Fatemi E, Khalili MA, Ghorbani R. Localized Application of Vibration Improves Passive Knee Extension in Women with Apparent Reduced Hamstring Extensibility: a Randomized Trial. J of physiotherapy 2011; 57:165-171.

6. Rauch F. Vibration Therapy. Developmental Medicine \& Child Neurology 2009; 51(suppl 4):166-168.

7. Schütz A. Klinischer Einsatz des V-Actor Handstücks zur Ergänzung der Stoßwellentherapie-Ein Erfahrungsbericht. Orthopädisches Nachricht 2008.

8. Mikhael M, Orr R, Amsen F, Greene D, Singh M. Effect of Standing Posture During Whole Body Vibration Training on Muscle Morophology and Function in Older Adults: A Randomized Controlled Trial. BMC Geriatrics 2010; 10(74):1-13.

9. Saxena A, Ramdath S, O'Halloran P, Gerdesmeyer, Gollwitzer H. Extra-Corporeal Pulsed-Activated Therapy ("EPAT" sound wave) for Achilles Tendinopathy: a Prospective Study. J Foot Ankle Surg 2011; 50(3):315-319.

10. Cacchio A, Rompe J, Furia J, Susi P, Santilli V, De Paulis F. Shockwave Therapy for the Treatment of Chronic Proximal Hamstring Tendinopathy in Professional Athletes. Am Journal of Sports medicine 2011; 39(1):146-152. 